\title{
Sleep problems and psychological symptoms as predictors of musculoskeletal conditions in children and adolescents
}

\author{
Alessandro Andreucci ${ }^{\mathrm{a}, \mathrm{b}}, \mathrm{PhD}$, Paul Campbell ${ }^{\mathrm{a}, \mathrm{c}}, \mathrm{PhD}$, Emily Richardson ${ }^{\mathrm{a}}$, Ying Chen ${ }^{\mathrm{a}, \mathrm{d}}$, \\ $\mathrm{PhD}$, Kate M Dunn ${ }^{\mathrm{a}} \mathrm{PhD}$
}

Running head: Childhood predictors of musculoskeletal conditions

\section{Affiliation:}

a Primary Care Centre Versus Arthritis, Research Institute for Primary Care \& Health Sciences, Keele University, Keele, Staffordshire, ST5 5BG, UK

${ }^{\mathrm{b}}$ Center for General Practice at Aalborg University, Department of Clinical Medicine, Aalborg University, Aalborg, Denmark

${ }^{c}$ Midlands Partnership NHS Foundation Trust, St Georges Hospital, Stafford, ST16 3SR, UK ${ }^{\mathrm{d}}$ Keele Clinical Trials Unit, David Weatherall Building, Keele University, Staffordshire, ST5 5BG, United Kingdom

\section{Corresponding author:}

Alessandro Andreucci, Center for General Practice at Aalborg University, Department of Clinical Medicine, Aalborg University, Aalborg, Denmark, aa@dcm.aau.dk

\section{Category}

Original article

\section{Funding source}

This article has been accepted for publication and undergone full peer review but has not been through the copyediting, typesetting, pagination and proofreading process, which may lead to differences between this version and the Version of Record. Please cite this article as doi: 10.1002/EJP.1491 
Dr. Alessandro Andreucci was funded by the Keele ACORN studentship. The creation and maintenance of the CiPCA dataset was funded by the Keele University Research Institute for Primary Care and Health Sciences (iPCHS) and the Primary Care Research Consortium.

Conflict of interest statement: The authors have no conflicts of interest relevant to this article to disclose.

\section{Significance}

Population-based studies showed that sleep problems and psychological symptoms may be important precursors of musculoskeletal pain in children and adolescents. By investigating these associations in primary care settings, this study showed that children and adolescents with consultations for sleep problems or psychological symptoms were at increased risk of subsequent consultations for musculoskeletal conditions. These findings support results from population-based studies, and identify potential areas for further research and potentially intervention within primary care.

\footnotetext{
Abbreviations

HR = Hazard Ratio

$\mathrm{CI}=$ Confidence Interval

CiPCA $=$ Consultations in Primary Care Archive

UK = United Kingdom
} 


\begin{abstract}
Background

Population-based studies show that sleep problems and psychological symptoms may increase the risk of musculoskeletal pain onset in children / adolescents. However, there is no evidence of these associations within primary care settings, where interventions can take place. This study investigated whether children / adolescents who consulted their general practitioner for sleep problems or psychological symptoms are at higher risk of subsequent consultations for musculoskeletal conditions.
\end{abstract}

\title{
Methods
}

This prospective matched-cohort study used electronic medical records of children / adolescents (aged 6-19 years) from a UK primary care database. Associations between a consultation for sleep problems or psychological symptoms at baseline and a subsequent consultation for musculoskeletal conditions within the two-year follow-up were investigated using survival analysis, producing Hazard Ratios (HR) and 95\% Confidence Intervals (CI) with adjustment for confounders.

\section{Results}

Children / adolescents who consulted for sleep problems had a significant increased risk of consultation for musculoskeletal conditions ( $\mathrm{HR}=1.72$; 95\% CI 1.14, 2.60), which became non-significant after adjustment for confounders ( $\mathrm{HR}=1.49 ; 95 \% \mathrm{CI} 0.98,2.27)$. Children / adolescents who consulted for psychological symptoms had a significant increased risk of consultation for musculoskeletal conditions $(\mathrm{HR}=1.59 ; 95 \% \mathrm{CI} 1.31,1.93)$, which was attenuated after adjustment $(\mathrm{HR}=1.39 ; 95 \% \mathrm{CI} 1.14,1.70)$.

\section{Conclusions}

Children and adolescents who visit primary care for sleep problems or psychological symptoms have increased risk of future musculoskeletal consultations. Further work is needed to understand the causal mechanisms that explain these associations, before designing interventions strategies within primary care settings. 


\section{Introduction}

Musculoskeletal pain in children and adolescents is common and burdensome; prevalence is high (ranging from 4 to $40 \%$ (King et al., 2011)) with conditions like low back pain and neck pain ranked in the top five globally (for ages 10 to 19 years) of years of life lived with disability (YLDs) (Mokdad et al., 2016). Two factors which are frequently associated with the onset of musculoskeletal conditions in the child/adolescent general population are sleep problems (Auvinen et al., 2010; Harrison et al., 2014; Ståhl et al., 2008) and psychological symptoms (Jones et al., 2003a, 2003b; Jussila et al., 2014). Both of these potential risk factors are common. It is estimated that $25-40 \%$ of children/adolescents experience sleep problems (e.g. bedtime problems, night wakings, sleep related anxiety, deficient sleep, and poor sleep hygiene) (Dosi et al., 2015; Meltzer et al., 2014) and approximately 33\% experience a psychological symptom (e.g. anxiety, depression, attention deficit hyperactivity disorder) (Merikangas et al., 2009). Proposed biological mechanisms for the pathway to the development of musculoskeletal pain from sleep problems or psychological symptoms include; cytokine and inflammatory mediators, impaired relaxation of the muscles, changes of pain thresholds, dysfunction of the hypothalamic-pituitary-adrenal (HPA) axis and the effect of mood/anxiety on pain thresholds (Auvinen et al., 2010; Christensen et al., 2019; Gatchel et al., 2007; Harrison et al., 2014; McBeth et al., 2007).

Research on sleep problems and psychological symptoms has shown associations with musculoskeletal pain in child/adolescent population-based studies (Auvinen et al., 2010; Harrison et al., 2014; Jones et al., 2003a, 2003b; Jussila et al., 2014; Ståhl et al., 2008), but the importance or impact of findings are unclear because the reported musculoskeletal pain is often mild or self-limiting. Researching these associations in clinical settings would naturally exclude the mildest cases, as the pain is, by definition, important enough to seek healthcare for. Whilst there is evidence that musculoskeletal pain, sleep problems, and psychological symptoms are common presentations in primary care for children/adolescents (Cornish et al., 2016; Henschke et al., 2014; Honaker and Meltzer, 2016; de Inocencio, 1998; De Inocencio, 2004; Jordan et al., 2010; Michaleff et al., 2017; van Suijlekom-Smit et al., 1997), no studies to date have examined their relationships. The aim of this study is to investigate whether consultations for sleep problems or psychological symptoms are associated with subsequent consultations for musculoskeletal pain in children/adolescents in primary care.

This article is protected by copyright. All rights reserved 


\section{Patients and Methods}

\section{Setting}

Data from the Consultations in Primary Care Archive (CiPCA) was used within this study. CiPCA is a database comprising medical consultation data (e.g. diagnoses, symptoms, prescriptions, referrals) recorded since 1998 from a sample of general practices in North Staffordshire, United Kingdom (UK) (Jordan et al., 2007, 2010; Porcheret et al., 2004). Previous studies have shown similar consultation prevalence rates of musculoskeletal conditions between CiPCA, UK national and European datasets, providing evidence for validity and generalisability (Jordan et al., 2007, 2014). To maintain data quality, an annual cycle of training in morbidity coding, assessment, and auditing is undertaken by participating CiPCA general practices (Jordan et al., 2010; Porcheret et al., 2004). CiPCA has ethical approval (North West - Haydock REC ref: 17/NW/0232) and data is pseudo anonymised (Jordan et al., 2010; Porcheret et al., 2004). The use of consultation data assures good representativeness of healthcare attendance in the general population, as more than $98 \%$ of the population in the UK are registered with a general practice (Herrett et al., 2015; Jordan et al., 2010).

\section{Design and cohort definition}

This is a prospective matched-cohort study nested within a medical record database. Medical record consultation data from 11 general practices, between 2005 and 2012, were used. Two analyses were carried out within this current study in order to explore the risk of having musculoskeletal consultations following consultations indicating two single distinct exposures: sleep problems and psychological symptoms. One dataset was created to investigate the relationship between consultations for sleep problems (exposure) and subsequent musculoskeletal consultations (outcome), the second dataset was used to investigate the relationship between consultations for psychological symptoms (exposure) and subsequent musculoskeletal consultations (outcome). However, in acknowledgement of potential overlap between these conditions within one person, the multivariable analysis models controlled for each other exposure accordingly. Each analysis used a matched cohort design (individuals with exposure at baseline vs. matched individuals without exposure). Each matched cohort dataset was created by selecting the individuals with a consultation for sleep problems or psychological symptoms between 2005 and 2010, respectively. These individuals were considered "exposed", and were assigned an "index date" (i.e. date of first 
consultation for the exposure) and matched with up to five controls ("unexposed individuals"). Individuals were matched on age at index date (up to \pm 2 years), gender and general practice, following previous methodology (Green et al., 2015; Muller et al., 2014). Matched individuals were assigned an "index date" that was equivalent to the date of first consultation for the exposure variable. Within each cohort, "exposed" individuals and selected "unexposed" controls were followed-up for a period of 2-years after the "index date".

\section{Participant inclusion criteria}

The study population included those patients who were continually registered at each practice over the study period 2005-2012 to ensure capturing active patients. Children and adolescents were included if they were aged between 6 and 19 years old (the age of 6 has been reported as the starting point for children to use the word "pain", and age 19 is defined by the World Health Organization as the end of adolescence) (von Baeyer, 2006; Stanford et al., 2005; World Health Organization, 2013) at "index date" (i.e. date of consultation for the exposure at baseline). Any adolescents who became older than 19 years during follow-up (i.e. 2 years after the first consultation for the exposure) were censored (i.e. only the time from the index date to the date when adolescents became 19 years old was entered in the analysis). To ensure incident status and avoid reversed association (i.e. musculoskeletal consultations leading to subsequent consultations for sleep problems or psychological symptoms), data was screened for a period of 2 years prior to the "index date" to check that neither those in the exposed group or control group had consulted about a musculoskeletal consultation. This ensured that individuals did not have a consultation for musculoskeletal pain at baseline, and therefore a consultation for a musculoskeletal condition post baseline would represent new onset. The matched "unexposed" individuals were also censored if they had a consultation for the exposure variable in the period after the index date and prior to a consultation for a musculoskeletal condition or the end of the follow-up period (i.e. only the time from the index date to the date when the "unexposed" individuals had a consultation for the exposure was entered in the analysis).

\section{Identification of variables}

Consultations for sleep problems (e.g. sleep disturbances, insomnia, nocturnal cough/wheeze, tired all the time), psychological symptoms (e.g. health anxiety, stress related problem, behaviour disorder, low mood, disturbance of conduct) and musculoskeletal pain (e.g. low 
back pain, cervicalgia, arthralgia of the knee), were identified by means of Read Codes (a list of the 5 most frequent Read Codes used in this study for each variable is outlined in Table 1). The Read Code system is used within the UK to electronically record all consultations and patient encounters in primary care. They are organised into hierarchical chapters following the International Classification of Disease (ICD-10) criteria (Benson, 2012). Thus, when an individual consult for a condition, the presenting symptom for that condition (e.g. insomnia) is recorded by means of a Read Code for that specific symptom (e.g. E2741). Read Codes used in this study were identified through reviewing previous relevant studies (a list of studies is available from the authors), and by including further Read Codes from lists previously used within the Research Institute of Primary Care and Health Sciences at Keele University (https://www.keele.ac.uk/mrr/morbiditydefinitions/). The identified Read Codes lists were checked for relevance and appropriateness for the condition that was to be identified (e.g. outcome, exposure, confounders) via consensus with an academic GP (E.R.) experienced in the application of Read Codes during consultations. All Read Codes used in this study are available on request to the corresponding author.

\section{Data analysis}

Descriptive analyses of the baseline variables for both datasets were carried out. Survival curves were displayed using Kaplan-Meier graphs, to describe the difference in survival. Survival analysis was carried out using Cox regression models (i.e. time to event analysis, one for each exposure) each with three stages. Stage 1 estimated the hazard ratio for a musculoskeletal consultation in consulters with the exposure compared to those without the exposure (i.e. an hazard ratio > 1 means that at any given point in time during the 2 -year follow-up, those with the exposure are at increased risk of musculoskeletal consultations compared to those without the exposure). The model at stage 2 was then adjusted by gender, age at index date, practice (to take into account potential inaccuracies that may have occurred when matching for gender, age and practice), index year and the inclusion of the other exposure (e.g. inclusion of psychological symptoms within the sleep model, and vice versa). Stage 3 of the model included a further adjustment for consultation frequency to account for surveillance bias. The proportional hazard assumption was tested by the Schoenfeld residuals test. When the test was significant, the variable for which the test was significant was entered in the analysis as a time-varying confounder following the methodology described previously (Bellera et al., 2010). Analysis was performed using STATA 14. 


\section{Results}

\section{Descriptive analysis}

Baseline descriptive analyses of both datasets are outlined in Table 2 and Table 3.

\section{Sleep problems - musculoskeletal conditions dataset}

The mean age was 13.1 years and there were 291 boys (45.6\%) and 347 girls (54.4\%) in the sleep exposure model dataset. The dataset included 107 children/adolescents ( 49 boys and 58 girls) who had a record for sleep problems, matched to 531 controls (242 boys and 289 girls) without such record (Table 2). Among the 638 children/adolescents, 123 (19.3\%; annual incidence rate 96/1000 individuals) consulted for a musculoskeletal condition within the 2year follow-up period (Table 3). The proportion of children/adolescents with a consultation for a musculoskeletal condition was higher among those with sleep problems compared to those without sleep problems ( $28.1 \%$ vs. $17.5 \%$; annual incidence rate $140 / 1000$ vs. $88 / 1000$ individuals, respectively) (Table 3).

\section{Psychological symptoms - musculoskeletal conditions dataset}

The mean age was 13.7 years and there were 1,476 boys (48.5\%) and 1,566 girls (51.5\%) within the psychological symptoms exposure model dataset. The dataset included 507 exposed children/adolescents ( 246 boys and 261 girls) who had a recorded psychological symptom, matched to 2,535 controls (1,230 boys and 1,305 girls) without such record (Table 2). Among the 3,042 children/adolescents, 574 (18.9\%; annual incidence rate 94/1000 individuals) consulted for a musculoskeletal condition within the 2-year follow-up period (Table 3). The proportion of children/adolescents with a consultation for a musculoskeletal condition was higher in those with psychological symptoms compared to those without psychological symptoms (26.0\% vs. $17.4 \%$; annual incidence rate $130 / 1000$ vs. $87 / 1000$ individuals, respectively) (Table 3).

\section{Survival analysis of the association between consultations for the exposure and consultations for musculoskeletal conditions}

Kaplan-Meier graphs 
Survival curves are presented in Figure 1 and Figure 2. Three control "unexposed" individuals $(0.6 \%)$ were censored because they had a consultation for sleep problems in the period after the index date and prior to a consultation for a musculoskeletal condition within the sleep exposure model dataset. Twenty-eight control "unexposed" individuals (1.1\%) were censored because they had a consultation for psychological symptoms in the period after the index date and prior to a consultation for a musculoskeletal condition within the psychological symptoms exposure model dataset.

\section{Survival analysis using Cox regression modelling}

Results of unadjusted Cox regression analysis of the association between sleep problems and consultation for musculoskeletal conditions (Table 4) show a statistically significant increased hazard (Hazard ratio $(\mathrm{HR})=1.72 ; 95 \%$ Confidence Interval $(\mathrm{CI}) 1.14,2.60)$ of consultation for a musculoskeletal condition in children/adolescents with sleep problems compared to those without (stage 1). This estimate was unchanged (HR $=1.72 ; 95 \%$ CI 1.13 , 2.60) after adjustment for potential confounders (stage 2), although was attenuated to a nonsignificant increased hazard $(\mathrm{HR}=1.49 ; 95 \%$ CI 0.98, 2.27) after further adjustment for consultation frequency (stage 3). Unadjusted Cox regression analysis in those with coded psychological symptoms (Table 5) show a statistically significant increased hazard $(\mathrm{HR}=$ $1.59 ; 95 \%$ CI $1.31,1.93$ ) of consultation for a musculoskeletal condition in exposed children/adolescents (stage 1). This estimate was unchanged (HR = 1.59; 95\% CI 1.31, 1.94) after adjustment for potential confounders (stage 2$)$, but was reduced further (HR = 1.39; $95 \%$ CI 1.14, 1.70) after adjustment for consultation frequency (stage 3 ). 


\section{Discussion}

This study provides new prospective evidence that children and adolescents seeking primary health care for sleep problems or psychological symptoms are at increased risk of later musculoskeletal pain consultations compared to those without sleep problems or psychological symptoms. There was increased hazard of $49 \%$ for a musculoskeletal consultation among children/adolescents who had consulted previously with sleep problems (annual incidence rate 140/1000 in those with sleep problems vs. 88/1000 in those without). Similarly, there was an increased hazard of 39\% among those who had previously consulted for psychological symptoms (annual incidence rate 130/1000 in those with psychological symptoms vs. 87/1000 in those without). These results support findings from populationbased studies, and suggest that an increased risk of musculoskeletal consultation among children/adolescents with sleep problems or psychological symptoms exists at a clinical level. Sleep problems and psychological symptoms may be important starting points for the onset of musculoskeletal pain in children and adolescents, and primary health care visits may be critical points for intervention to prevent musculoskeletal pain onset.

\section{Comparison with previous literature}

There is currently no other literature that investigated the association between sleep problems, psychological symptoms and musculoskeletal pain within primary care settings for children/adolescents, but comparisons can be drawn from the evidence within populationbased samples. A recent systematic review of 13 studies concludes that sleep problems are overall not risk factors for the onset of musculoskeletal pain in children or adolescents (Andreucci et al., 2017), as opposed by evidence from studies conducted in adults (Gupta et al., 2007; McBeth et al., 2014; Nitter et al., 2012). However the trends of risk within the systematic review showed elevated risk in the presence of sleep problems, which is in accord with the findings of this study (i.e. non-significant increased hazard). Similarly, evidence shows that in child/adolescent (Jones et al., 2003a, 2003b) and in adult populations (McBeth and Jones, 2007; Pinheiro et al., 2015), psychological symptoms are predictive of future musculoskeletal pain onset, and this study has demonstrated an increased risk within a primary care consultation population. The results found in this current study may also have been influenced by the consultation process. Children/adolescents who present to primary care may be characterized by a higher level of severity, greater frequency and longer duration 
of musculoskeletal conditions (Campbell and Roland, 1996) than children and adolescents reporting pain in the general population. In effect musculoskeletal pain might have preceded the consultation for the exposure, and this experience could have initiated a reciprocal relationship between exposure and musculoskeletal pain. For example, there is a well evidenced reciprocal relationship between sleep and pain (Christensen et al., 2019; Finan et al., 2013; McBeth et al., 2015), where pain disrupts sleep, which can then impact on the experience of pain; this may have increased the severity of both symptoms leading to a higher probability of consultation. For the influence of psychological symptoms, the diathesis stress model (Dersh et al., 2002) suggests that latent psychological symptoms can elevate stress levels that then moderate the experience of future adverse events (e.g. a musculoskeletal injury) which may in turn worsen the psychological status of the individual and exacerbate the experience of pain leading to healthcare seeking. This therefore illustrates the need for additional research within child and adolescent consultation populations to ascertain the starting points of the actual experience (of exposures and outcomes) as well as the evidence of when consultation occurs.

\section{Strengths and weaknesses}

This study has a number of strengths. First, analysis was performed using prospectively collected routine data from a large and high-quality primary care dataset, which allows the use of the entire temporal frame of data compared to studies where data are collected solely at fixed points in time. In addition, the dataset includes actual "real" consultation events, therefore limiting the occurrence of selection bias or reporting bias from participants. Second, in order to reduce the risk of confounding, individuals were matched by variables that may be associated with increased healthcare seeking and musculoskeletal pain, such as age, gender and practice (Campbell and Roland, 1996; Henschke et al., 2015; Kamper et al., 2016; King et al., 2011; McBeth and Jones, 2007) and the analysis was also adjusted for consultation frequency, to control for a potential increased probability of consultation for exposure and/or outcome by virtue of increased consultations (surveillance bias). Third, the results are generalizable to the general population, as $98 \%$ of the UK population is registered with a GP, and CiPCA has a similar prevalence of musculoskeletal consultations when compared with national and international databases (Herrett et al., 2015; Jordan et al., 2007, 2010, 2014). This current study also includes several limitations. First, limitations related to the use of 
Read Codes, such as the inability to assess the severity, duration and impact of pain or the cause of the condition (Michaleff et al., 2017; Muller, 2014). There is also the inability to adjust for variables relative to lifestyle (e.g. socioeconomic status, BMI, smoking, physical activity, substance use) which may have been important (Kamper et al., 2018), as such information is generally less well recorded within clinical coding systems (Glasgow et al., 2012). In addition many children/adolescents may have had pre-clinical status for both exposure and outcome (which may have included individuals in the matched controls) (Glasgow et al., 2012; Jordan et al., 2010; Muller, 2014). Also there are potential issues which may affect data quality, such as comorbidities that are not recorded at the time of consultation, as often only the most prominent or most recent complaint would be recorded (Jordan and Croft, 2008; Muller, 2014). For example, a patient with a long-term musculoskeletal pain condition who recently consulted about sleep problems may only have sleep problems recorded. Second, whilst this study endeavored to account for "incident" musculoskeletal pain consultations, and ensured that no musculoskeletal pain consultations occurred during the two years previous to the exposure (sleep problems, psychological symptoms) for each included patient, it is possible that children and adolescents within the study may have had musculoskeletal pain previous to that time period. In addition, other comorbidities (e.g., physical illnesses, chronic medical conditions) that might co-occur with psychological and sleep problems were not controlled for. Third, the decision to consult at primary care may be influenced by factors not measurable using electronic health records, (e.g. parent's perceived severity of symptoms, family patterns of illness behaviour, parental economic status, family size, perceived benefits of seeking care) (Campbell and Roland, 1996; Cardol et al., 2006, 2007). Fourth, over-adjustment (e.g. the variable for which the analysis is adjusted for is in the pathway between the exposure and the outcome and adjustment may bias the results towards the null) (Schisterman et al., 2009) might explain the attenuation of estimates after adjustment for consultation frequency (i.e. stage 2 to stage 3 change in both analysis models). Fifth, the dataset only included individuals continually registered between 2005-2012. Therefore, the data does not include those who have moved to another area or moved into the area. Research shows that children and adolescents with increased residential mobility are less likely to flourish and are at higher risk of psychological symptoms (Gjelsvik et al., 2019) and therefore some bias may have been introduced into the results. Finally, this study used data from the primary care system in the UK, therefore results may not be generalizable to other healthcare systems with different models of care (Kringos et al., 2013).

This article is protected by copyright. All rights reserved 


\section{Implications}

This study showed an increased risk of seeking primary health care for musculoskeletal pain in children/adolescents who had previously sought primary health care for sleep problems or psychological symptoms. Whilst the size of estimates reported are modest in terms of the increase in risk to an individual, the evidence of the relatively high prevalence of sleep problems, psychological symptoms, and musculoskeletal pain, within this population (Cornish et al., 2016; Henschke et al., 2014; Honaker and Meltzer, 2016; De Inocencio, 2004; Jordan et al., 2010; Meltzer et al., 2010; Michaleff et al., 2017; van Suijlekom-Smit et al., 1997) highlights the importance at a population level. Routine screening in primary care settings for the detection of children/adolescents with sleep problems and/or with psychological symptoms for early recognition of symptoms may be performed, and information and education given about potential risks as a pre-emptive intervention (Kramer and Garralda, 2000; Meltzer et al., 2014). Similarly, children/adolescents who present to primary care with musculoskeletal problems might benefit from education on sleep hygiene, or on how to cope with pain and this might prevent the transition to chronicity and worsening of musculoskeletal problems (Vriend and Corkum, 2011). In addition, this study aimed to investigate whether consultations for sleep problems or psychological symptoms were associated with consultations for musculoskeletal pain and not to prove causation (Hernán, 2018), and only some causality criteria (e.g. temporality, consistency with previous research) but not others (e.g. biological gradient, experimental evidence) (Bhopal, 2002) were fulfilled. Therefore, the results of this study are a first step to understanding these associations and primarily there is a need to understand the mechanisms that lead to increased risk, in order to design effective interventions. Also, there is a need to conduct long-term longitudinal research to establish if these risk factors play a role in the development of long-term adult musculoskeletal pain.

\section{Conclusions}

This study found an increased risk of consultation for musculoskeletal conditions among children/adolescents with previous consultations for sleep problems or psychological symptoms. Future studies are needed to elucidate the causal mechanisms between these risk factors and musculoskeletal conditions, which might lay the basis for potential interventions within primary care settings. 


\section{Acknowledgments}

We would like to thank the Informatics team at the Institute for Primary Care \& Health Sciences (iPCHS) for providing technical support with the CiPCA dataset.

\section{Author contributions:}

Dr. Alessandro Andreucci conceptualized and designed the study, carried out the analyses, drafted the initial manuscript, and critically reviewed and revised the manuscript for important intellectual content. Dr. Paul Campbell and Prof. Kate M Dunn provided substantial contribution to the design of the study, and critically reviewed and revised the manuscript for important intellectual content. Dr. Ying Chen provided substantial contribution to the design of the analysis, to the interpretation of data, cleaned the data and prepared the cohorts from raw medical records according to the study design and critically reviewed and revised the manuscript for important intellectual content. Dr. Emily Richardson provided substantial contribution to the design of the study and the selection of variables for the analysis, and critically reviewed and revised the manuscript for important intellectual content. All authors approved the final manuscript as submitted and agree to be accountable for all aspects of the work. 


\section{References}

Andreucci, A., Campbell, P., Dunn, K.M. (2017). Are sleep problems a risk factor for the onset of musculoskeletal pain in children and adolescents? A systematic review. Sleep 40,7,111.

Auvinen, J.P., Tammelin, T.H., Taimela, S.P., Zitting, P.J., Jarvelin, M.R., Taanila, A.M., Karppinen, J.I. (2010). Is insufficient quantity and quality of sleep a risk factor for neck, shoulder and low back pain? A longitudinal study among adolescents. Eur Spine J 19, 641649.

von Baeyer, C.L. (2006). Children's self-reports of pain intensity: scale selection, limitations and interpretation. Pain Res Manag 11, 157-162.

Bellera, C.A., MacGrogan, G., Debled, M., de Lara, C.T., Brouste, V., Mathoulin-Pélissier, S. (2010). Variables with time-varying effects and the Cox model: some statistical concepts illustrated with a prognostic factor study in breast cancer. BMC Med Res Methodol 10, 20. Benson, T. (2012). The history of the Read Codes: The inaugural James Read memorial lecture 2011. Inform Prim Care 19, 173-182.

Bhopal, R. (2002). Concepts of Epidemiology - an integrated introduction to the ideas, theories, principles and methods of epidemiology (Oxford University Press).

Campbell, S.M., Roland, M.O. (1996). Why do people consult the doctor? Fam Pract 13, 7583.

Cardol, M., van Dijk, L., van den Bosch, W.J.H.M., Spreeuwenberg, P., de Bakker, D.H., Groenewegen, P.P. (2007). Striking variations in consultation rates with general practice reveal family influence. BMC Fam Pract 8, 4.

Cardol, M., Groenewegen, P.P., Spreeuwenberg, P., Van Dijk, L., Van Den Bosch, W.J.H.M., De Bakker, D.H. (2006). Why does it run in families? Explaining family similarity in help-seeking behaviour by shared circumstances, socialisation and selection. Soc Sci Med 63, 920-932.

Christensen, J., Noel, M., Mychasiuk, R. (2019). Neurobiological mechanisms underlying the sleep-pain relationship in adolescence: A review. Neurosci Biobehav Rev 96, 401-413. Cornish, R.P., John, A., Boyd, A., Tilling, K., Macleod, J. (2016). Defining adolescent common mental disorders using electronic primary care data: a comparison with outcomes measured using the CIS-R. BMJ Open 6, e013167.

Dersh, J., Polatin, P.B., Gatchel, R.J. (2002). Chronic Pain and Psychopathology: Research 
Findings and Theoretical Considerations. Psychosom Med 64, 773-786.

Dosi, C., Figura, M., Ferri, R., Bruni, O. (2015). Sleep and Headache. Semin Pediatr Neurol $22,105-112$.

Finan, P.H., Goodin, B.R., Smith, M.T. (2013). The association of sleep and pain: An update and a path forward. J Pain 14, 1539-1552.

Gatchel, R.J., Peng, Y.B., Peters, M.L., Fuchs, P.N., Turk, D.C. (2007). The biopsychosocial approach to chronic pain: Scientific advances and future directions. Psychol Bull 133, 581624.

Gjelsvik, A., Rogers, M.L., Song, L., Field, A.E., Vivier, P.M. (2019). Residential Mobility and Flourishing Among United States School-Age Children, 2011/2012 National Survey of Children's Health. Matern Child Health J 23, 522-529.

Glasgow, R.E., Kaplan, R.M., Ockene, J.K., Fisher, E.B., Emmons, K.M. (2012). Patientreported measures of psychosocial issues and health behavior should be added to electronic health records. Health Aff 31, 497-504.

Green, D., Muller, S., Mallen, C., Hider, S. (2015). Fatigue as a precursor to polymyalgia rheumatica: an explorative retrospective cohort study. Scand J Rheumatol 44, 219-223. Gupta, A., Silman, A.J., Ray, D., Morriss, R., Dickens, C., MacFarlane, G.J., Chiu, Y.H., Nicholl, B., McBeth, J. (2007). The role of psychosocial factors in predicting the onset of chronic widespread pain: results from a prospective population-based study. Rheumatology (Oxford) 46, 666-671.

Harrison, L., Wilson, S., Munafo, M.R. (2014). Exploring the associations between sleep problems and chronic musculoskeletal pain in adolescents: A prospective cohort study. Pain Res Manag 19, e139-e145.

Henschke, N., Harrison, C., McKay, D., Broderick, C., Latimer, J., Britt, H., Maher, C.G. (2014). Musculoskeletal conditions in children and adolescents managed in Australian primary care. BMC Musculoskelet Disord 15, 164.

Henschke, N., Kamper, S.J., Maher, C.G. (2015). The Epidemiology and Economic Consequences of Pain. Mayo Clin Proc 90, 139-147.

Hernán, M.A. (2018). The C-word: Scientific euphemisms do not improve causal inference from observational data. Am J Public Health 108, 616-619.

Herrett, E., Gallagher, A.M., Bhaskaran, K., Forbes, H., Mathur, R., Staa, T. van, Smeeth, L. (2015). Data Resource Profile: Clinical Practice Research Datalink (CPRD). Int J Epidemiol 44, 827-836.

Honaker, S.M., Meltzer, L.J. (2016). Sleep in pediatric primary care: A review of the 
literature. Sleep Med Rev 25, 31-39.

de Inocencio, J. (1998). Musculoskeletal pain in primary pediatric care: analysis of 1000 consecutive general pediatric clinic visits. Pediatrics 102, E63.

De Inocencio, J. (2004). Epidemiology of musculoskeletal pain in primary care. Arch Dis Child 89, 431-434.

Jones, G.T., Silman, A.J., Macfarlane, G.J. (2003a). Predicting the onset of widespread body pain among children. Arthritis Rheum 48, 2615-2621.

Jones, G.T., Watson, K.D., Silman, A.J., Symmons, D.P., Macfarlane, G.J. (2003b).

Predictors of low back pain in British schoolchildren: a population-based prospective cohort study. Pediatrics 111, 822-828.

Jordan, K., Clarke, A.M., Symmons, D.P.M., Fleming, D., Porcheret, M., Kadam, U.T., Croft, P. (2007). Measuring disease prevalence: A comparison of musculoskeletal disease using four general practice consultation databases. Br J Gen Pract 57, 7-14.

Jordan, K.P., Croft, P. (2008). Opportunities and limitations of general practice databases in pain research. 137, 469-470.

Jordan, K.P., Jöud, A., Bergknut, C., Croft, P., Edwards, J.J., Peat, G., Petersson, I.F., Turkiewicz, A., Wilkie, R., Englund, M. (2014). International comparisons of the consultation prevalence of musculoskeletal conditions using population-based healthcare data from England and Sweden. Ann Rheum Dis 73, 212-218.

Jordan, K.P., Kadam, U.T., Hayward, R., Porcheret, M., Young, C., Croft, P. (2010). Annual consultation prevalence of regional musculoskeletal problems in primary care: an observational study. BMC Musculoskelet Disord 11, 144.

Jussila, L., Paananen, M., Nayha, S., Taimela, S., Tammelin, T., Auvinen, J., Karppinen, J. (2014). Psychosocial and lifestyle correlates of musculoskeletal pain patterns in adolescence: A 2-year follow-up study. Eur J Pain 18, 139-146.

Kamper, S.J., Henschke, N., Hestbaek, L., Dunn, K.M., Williams, C.M. (2016).

Musculoskeletal pain in children and adolescents. Braz J Phys Ther 1-10.

Kamper, S.J., Michaleff, Z.A., Campbell, P., Dunn, K.M., Yamato, T.P., Hodder, R.K., Wiggers, J., Williams, C.M. (2018). Back pain, mental health and substance use are associated in adolescents. J Public Health (Bangkok) 1-7.

King, S., Chambers, C.T., Huguet, A., MacNevin, R.C., McGrath, P.J., Parker, L., MacDonald, A.J. (2011). The epidemiology of chronic pain in children and adolescents revisited: a systematic review. Pain 152, 2729-2738.

Kramer, T., Garralda, M.E. (2000). Child and adolescent mental health problems in primary 
care. Adv Psychiatr Treat 6, 287-294.

Kringos, D., Boerma, W., Bourgueil, Y., Cartier, T., Dedeu, T., Hasvold, T., Hutchinson, A., Lember, M., Oleszczyk, M., Rotar Pavlic, D., Svab, I., Tedeschi, P., Wilm, S., Wilson, A., Windak, A., Van Der Zee, J., Groenewegen, P. (2013). The strength of primary care in Europe: An international comparative study. Br J Gen Pract 63, 742-750.

McBeth, J., Jones, K. (2007). Epidemiology of chronic musculoskeletal pain. Best Pract Res Rheumatol 21, 403-425.

McBeth, J., Lacey, R.J., Wilkie, R. (2014). Predictors of new-onset widespread pain in older adults: results from a population-based prospective cohort study in the UK. Arthritis Rheumatol (Hoboken, NJ) 66, 757-767.

McBeth, J., Silman, A.J., Gupta, A., Chiu, Y.H., Ray, D., Morriss, R., Dickens, C., King, Y., Macfarlane, G.J. (2007). Moderation of psychosocial risk factors through dysfunction of the hypothalamic-pituitary-adrenal stress axis in the onset of chronic widespread musculoskeletal pain: Findings of a population-based prospective cohort study. Arthritis Rheum 56, 360-371. McBeth, J., Wilkie, R., Bedson, J., Chew-Graham, C., Lacey, R.J. (2015). Sleep Disturbance and Chronic Widespread Pain. Curr Rheumatol Rep 17.

Meltzer, L.J., Johnson, C., Crosette, J., Ramos, M., Mindell, J.A. (2010). Prevalence of Diagnosed Sleep Disorders in Pediatric Primary Care Practices. 125, 1410-1418.

Meltzer, L.J., Plaufcan, M.R., Thomas, J.H., Mindell, J.A. (2014). Sleep problems and sleep disorders in pediatric primary care: treatment recommendations, persistence, and health care utilization. J Clin Sleep Med 10, 421-426.

Merikangas, K.R., Nakamura, E.F., Kessler, R.C. (2009). Epidemiology of mental disorders in children and adolescents. Dialogues Clin Neurosci 11, 7-20.

Michaleff, Z.A., Campbell, P., Protheroe, J., Rajani, A., Dunn, K.M. (2017). Consultation patterns of children and adolescents with knee pain in UK general practice: analysis of medical records. BMC Musculoskelet Disord 18, 239.

Mokdad, A.H., Forouzanfar, M.H., Daoud, F., Mokdad, A.A., El Bcheraoui, C., MoradiLakeh, M., Kyu, H.H., Barber, R.M., Wagner, J., Cercy, K., Kravitz, H., Coggeshall, M., Chew, A., O’Rourke, K.F., Steiner, C., Tuffaha, M., Charara, R., Al-Ghamdi, E.A., Adi, Y., Afifi, R.A., Alahmadi, H., AlBuhairan, F., Allen, N., AlMazroa, M., Al-Nehmi, A.A., AlRayess, Z., Arora, M., Azzopardi, P., Barroso, C., Basulaiman, M., Bhutta, Z.A., Bonell, C., Breinbauer, C., Degenhardt, L., Denno, D., Fang, J., Fatusi, A., Feigl, A.B., Kakuma, R., Karam, N., Kennedy, E., Khoja, T.A.M., Maalouf, F., Obermeyer, C.M., Mattoo, A., McGovern, T., Memish, Z.A., Mensah, G.A., Patel, V., Petroni, S., Reavley, N., Zertuche, 
D.R., Saeedi, M., Santelli, J., Sawyer, S.M., Ssewamala, F., Taiwo, K., Tantawy, M., Viner, R.M., Waldfogel, J., Zu??iga, M.P., Naghavi, M., Wang, H., Vos, T., Lopez, A.D., Al Rabeeah, A.A., Patton, G.C., Murray, C.J.L. (2016). Global burden of diseases, injuries, and risk factors for young people's health during 1990???2013: a systematic analysis for the Global Burden of Disease Study 2013. Lancet 387, 2383-2401.

Muller, S. (2014). Electronic medical records: The way forward for primary care research? Fam Pract 31, 127-129.

Muller, S., Hider, S.L., Belcher, J., Helliwell, T., Mallen, C.D. (2014). Is cancer associated with polymyalgia rheumatica? A cohort study in the General Practice Research Database. Ann Rheum Dis 73, 1769-1773.

Nitter, A.K., Pripp, A.H., Forseth, K. (2012). Are sleep problems and non-specific health complaints risk factors for chronic pain? A prospective population-based study with 17 year follow-up. Scand J Pain 3, 210-217.

Pinheiro, M.B., Ferreira, M.L., Refshauge, K., Ordonana, J.R., Machado, G.C., Prado, L.R., Maher, C.G., Ferreira, P.H. (2015). Symptoms of Depression and Risk of New Episodes of Low Back Pain: A Systematic Review and Meta-Analysis. Arthritis Care Res (Hoboken) 67, 1591-1603.

Porcheret, M., Hughes, R., Evans, D., Jordan, K., Whitehurst, T., Ogden, H., Croft, P. (2004). Data Quality of General Practice Electronic Health Records: The Impact of a Program of Assessments, Feedback, and Training. J Am Med Informatics Assoc 11, 78-86.

Schisterman, E.F., Coleb, S.R., Platt, R.W. (2009). Overadjustment Bias and Unnecessary Adjustment in Epidemiologic Studies. 20, 488-495.

Ståhl, M., Kautiainen, H., El-Metwally, A., Häkkinen, A., Ylinen, J., Salminen, J.J., Mikkelsson, M. (2008). Non-specific neck pain in schoolchildren: Prognosis and risk factors for occurrence and persistence. A 4-year follow-up study. Pain 137, 316-322.

Stanford, E.A., Chambers, C.T., Craig, K.D. (2005). A normative analysis of the development of pain-related vocabulary in children. Pain 114, 278-284.

van Suijlekom-Smit, L.W., Bruijnzeels, M.A., van der Wouden, J.C., van der Velden, J., Visser, H.K., Dokter, H.J. (1997). Children referred for specialist care: a nationwide study in Dutch general practice. Br J Gen Pract 47, 19-23.

Vriend, J., Corkum, P. (2011). Clinical management of behavioral insomnia of childhood. Psychol Res Behav Manag 4, 69-79.

World Health Organization (2013). WHO - Definition of key terms. 
Figure 1. Association between consultation for sleep problems and musculoskeletal conditions. Explanation of the graph: At 2-year follow-up, 96/531 (18.1\%) individuals without sleep problems and 30/107 (28.0\%) consulters with sleep problems consulted for a musculoskeletal condition or were censored from the study, respectively.

\section{Figure 2. Association between consultation for psychological symptoms and} musculoskeletal conditions. Explanation of the graph: At 2-year follow-up, 470/2535 $(18.5 \%)$ individuals without psychological symptoms and 132/507 (26.0\%) consulters with psychological symptoms consulted for a musculoskeletal condition or were censored from the study, respectively. 
Table 1 Most frequent Read Codes for the variables used in the study

\section{Sleep problems $($ Total $\mathbf{N}=107)$}

\begin{tabular}{llll}
\hline Read Code term & Frequency & Percentage \\
\hline E205 - Tired all the time & 25 & $23.4 \%(25 / 107)$ \\
\hline R005 - (D) Sleep disturbances & 20 & $18.7 \%(20 / 107)$ \\
\hline 173B - Nocturnal cough/wheeze & 15 & $14.0 \%(15 / 107)$ \\
\hline R0050 - (D) Sleep disturbance unspecified & 13 & $12.1 \%(13 / 107)$ \\
\hline R0052 - (D) Insomnia NOS & 6 & $5.6 \%(6 / 107)$ \\
\hline
\end{tabular}

Psychological symptoms (Total $\mathrm{N}=507$ )

\begin{tabular}{llll}
\hline Read Code term & Frequency & Percentage \\
\hline ZV655 - (V) Health anxiety & 41 & $8.0 \%(41 / 507)$ \\
\hline E2C - Behaviour disorder & 40 & $7.9 \%(40 / 507)$ \\
\hline 1B1L - Stress related problem & 29 & $5.7 \%(29 / 507)$ \\
\hline 1BT - Low mood & 25 & $4.9 \%(25 / 507)$ \\
\hline 13Z4C - Behavioural problems at school & 19 & $3.8 \%(19 / 507)$ \\
\hline E2C - Disturbance of conduct NEC & 19 & $3.8 \%(19 / 507)$ \\
\hline
\end{tabular}

Musculoskeletal conditions (Total $\mathrm{N}=627$ )

\begin{tabular}{llll}
\hline Read Code term & Frequency & Percentage \\
\hline N145 - Back pain unspecified & 30 & $4.8 \%(30 / 627)$ \\
\hline N094M - Arthralgia of the knee & 28 & $4.5 \%(28 / 627)$ \\
\hline R065 - (D) Chest pain & 26 & $4.2 \%(26 / 627)$ \\
\hline N142 - Low back pain & 25 & $4.0 \%(25 / 627)$ \\
\hline N131 - Cervicalgia - pain in neck & 22 & $3.5 \%(22 / 627)$
\end{tabular}




\section{Table 2 Baseline descriptive analysis}

\begin{tabular}{llll}
\hline Sleep problems & Boys & Girls & Overall \\
\hline Age & 12.5 & 13.5 & 13.1 \\
\hline Individuals with sleep problems & $49(45.8 \%)$ & $58(54.2 \%)$ & 107 \\
Individuals without sleep problems & $242(45.6 \%)$ & $289(54.4 \%)$ & 531 \\
\hline Overall & $291(45.6 \%)$ & $347(54.4 \%)$ & \multicolumn{2}{l}{638} \\
\hline Psychological symptoms & Boys & Girls & Overall \\
\hline Age & 13.1 & 14.2 & 13.7 \\
\hline Individuals with psychological symptoms & $246(48.5 \%)$ & $261(51.5 \%)$ & 507 \\
Individuals without psychological symptoms & $1,230(48.5 \%)$ & $1,305(51.5 \%)$ & 2,535 \\
\hline Overall & $1,476(48.5 \%)$ & $1,566(51.5 \%)$ & 3,042 \\
\hline
\end{tabular}




\section{Table 3 Musculoskeletal pain frequency}

\section{Sleep problems and onset of musculoskeletal conditions}

\begin{tabular}{l|l|l|l}
\hline Consultation for musculoskeletal conditions & No & Yes & Overall \\
\hline Individuals with sleep problems & $77(71.9 \%)$ & $30(28.1 \%)$ & 107 \\
Controls without sleep problems & $438(82.5 \%)$ & $93(17.5 \%)$ & 531 \\
\hline Overall & $515(80.7 \%)$ & $123(19.3 \%)$ & 638 \\
\hline
\end{tabular}

Psychological symptoms and onset of musculoskeletal conditions

\begin{tabular}{l|l|l|l}
\hline Consultation for musculoskeletal conditions & No & Yes & Overall \\
\hline Individuals with psychological symptoms & $375(74.0 \%)$ & $132(26.0 \%)$ & 507 \\
Controls without psychological symptoms & $2,093(82.6 \%)$ & $442(17.4 \%)$ & 2,535 \\
\hline Overall & $2,468(81.1 \%)$ & $574(18.9 \%)$ & 3,042 \\
\hline
\end{tabular}


Table 4 Cox regression of the association between consultations for sleep problems at baseline and consultations for musculoskeletal conditions at follow-up

\begin{tabular}{l|c|c}
\hline Unadjusted analysis (Model 1) $(\mathbf{N}=\mathbf{6 3 8})$ & Hazard ratio & $\mathbf{9 5 \%}$ CI \\
\hline Musculoskeletal conditions at follow-up & 1.72 & $1.14,2.60$ \\
\hline Sleep problems &
\end{tabular}

Adjusted analysis ${ }^{\text {a }}$ (Model 2) $(\mathrm{N}=638)$

\begin{tabular}{l|c|c}
\hline Musculoskeletal conditions at follow-up & Hazard ratio & $\mathbf{9 5 \%} \mathbf{C I}$ \\
\hline Sleep problems & 1.72 & $1.13,2.60$ \\
\hline
\end{tabular}

Adjusted analysis ${ }^{\mathrm{b}}$ (Model 3) $(\mathrm{N}=638)$

Musculoskeletal conditions at follow-up

Sleep problems

\begin{tabular}{|c|c|} 
Hazard ratio & $\mathbf{9 5 \%} \mathbf{C I}$ \\
\hline 1.49 & $0.98,2.27$
\end{tabular}

${ }^{\mathrm{a}}$ Analysis adjusted for Psychological symptoms, Index year, Gender, Age at index date and Practice

${ }^{\mathrm{b}}$ Analysis adjusted for the above confounders and additionally by consultation frequency 
Table 5 Cox regression of the association between consultations for psychological symptoms at baseline and consultations for musculoskeletal conditions at follow-up

Unadjusted analysis (Model 1) $(\mathrm{N}=3,042)$

\begin{tabular}{l|c|c}
\hline Musculoskeletal conditions at follow-up & Hazard ratio & $\mathbf{9 5 \%}$ CI \\
\hline Psychological symptoms & 1.59 & $1.31,1.93$ \\
\hline
\end{tabular}

Adjusted analysis ${ }^{\mathrm{a}}($ Model 2$)(\mathrm{N}=3,042)$

\begin{tabular}{l|c|c}
\hline Musculoskeletal conditions at follow-up & Hazard ratio & $\mathbf{9 5 \%}$ CI \\
\hline Psychological symptoms & 1.59 & $1.31,1.94$ \\
\hline
\end{tabular}

Adjusted analysis ${ }^{\mathrm{b}}$ (Model 3) $(\mathrm{N}=\mathbf{3 , 0 4 2})$

\begin{tabular}{l|c|c|}
\hline Musculoskeletal conditions at follow-up & Hazard ratio & $\mathbf{9 5 \%}$ CI \\
\hline Psychological symptoms & 1.39 & $1.14,1.70$ \\
\hline
\end{tabular}

${ }^{a}$ Analysis adjusted for Sleep problems, Index year, Gender, Age at index date and Practice

${ }^{\mathrm{b}}$ Analysis adjusted for the above confounders and additionally by consultation frequency 
Figure 1

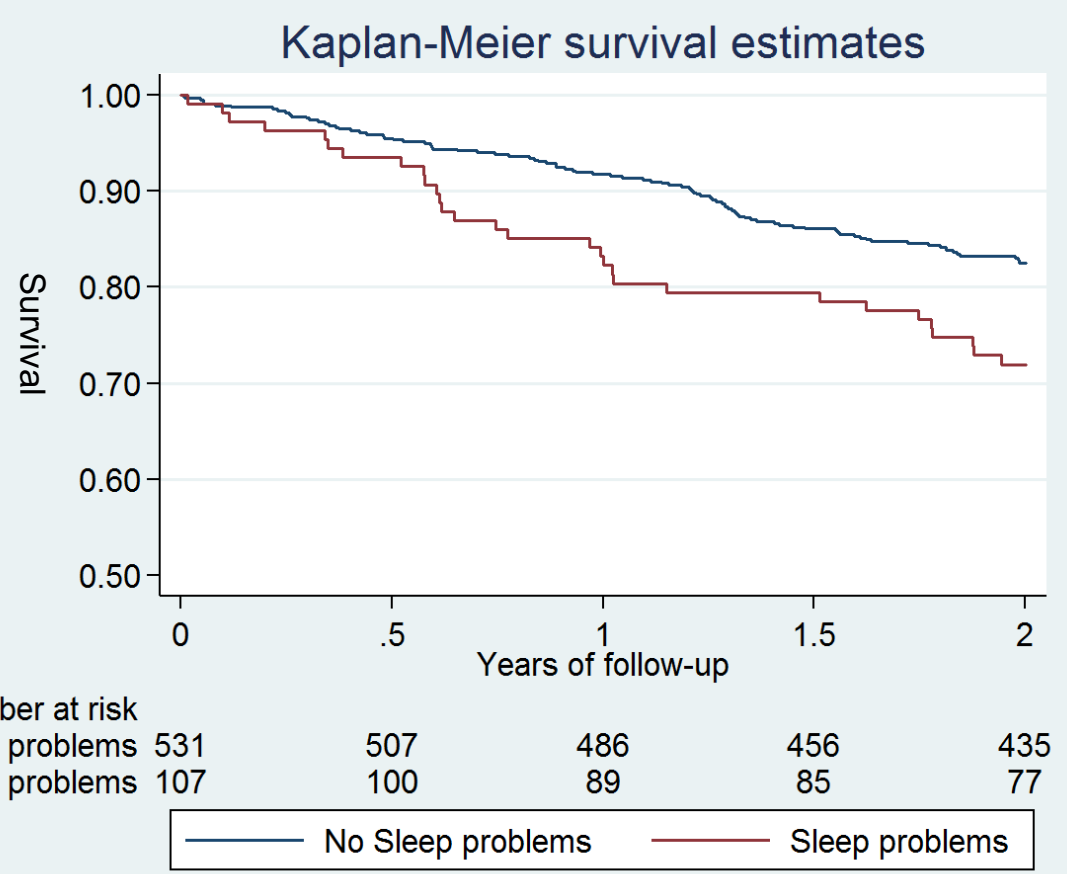

This article is protected by copyright. All rights reserved 
Figure 2

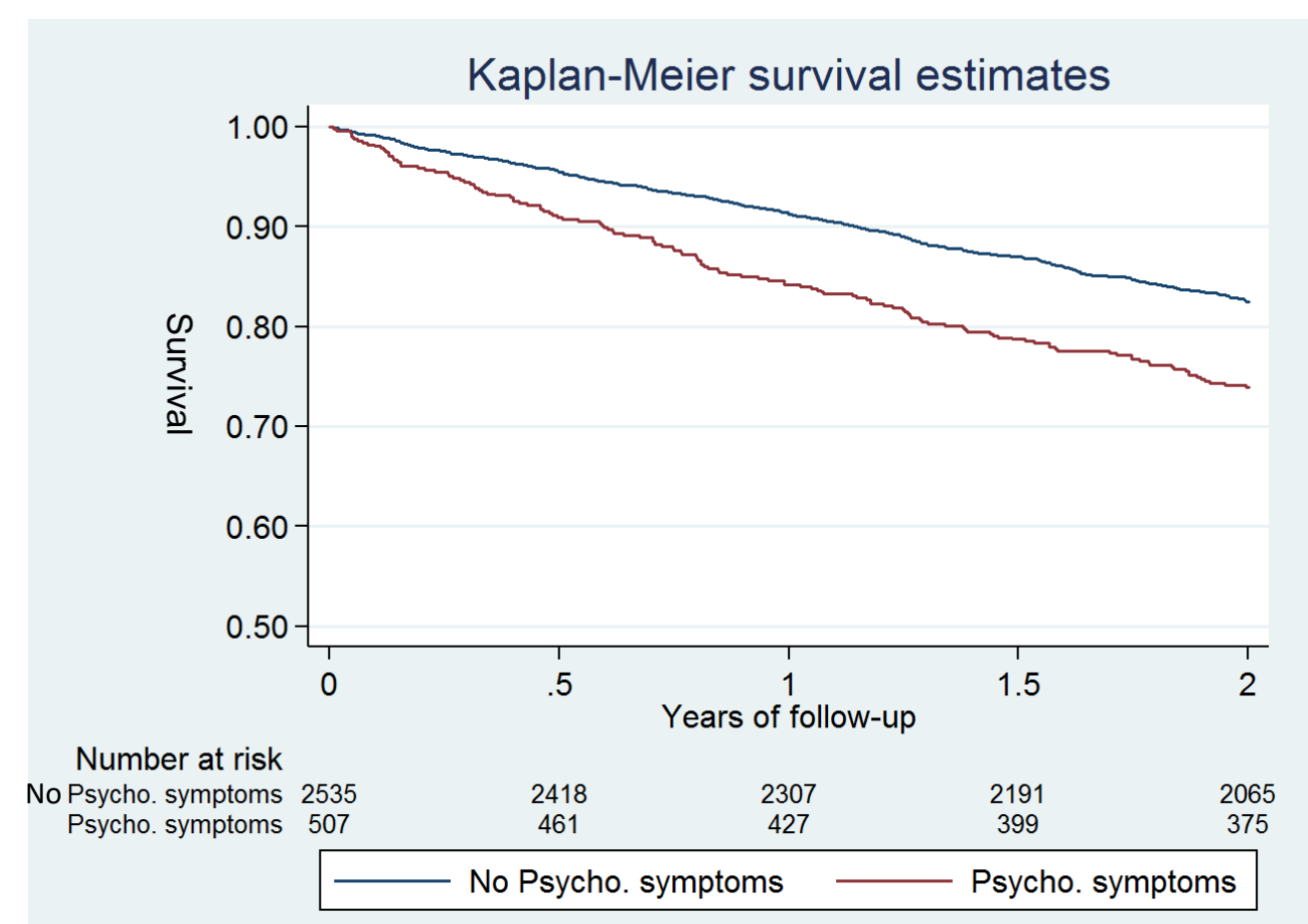

This article is protected by copyright. All rights reserved 